\title{
Digital Implementation of a Biological Astrocyte Model and Its Application
}

\author{
Hamid Soleimani, Mohammad Bavandpour, Arash Ahmadi, Member, IEEE, and Derek Abbott, Fellow, IEEE
}

\begin{abstract}
This paper presents a modified astrocyte model that allows a convenient digital implementation. This model is aimed at reproducing relevant biological astrocyte behaviors, which provide appropriate feedback control in regulating neuronal activities in the central nervous system. Accordingly, we investigate the feasibility of a digital implementation for a single astrocyte and a biological neuronal network model constructed by connecting two limit-cycle Hopf oscillators to an implementation of the proposed astrocyte model using oscillatorastrocyte interactions with weak coupling. Hardware synthesis, physical implementation on field-programmable gate array, and theoretical analysis confirm that the proposed astrocyte model, with considerably low hardware overhead, can mimic biological astrocyte model behaviors, resulting in desynchronization of the two coupled limit-cycle oscillators.
\end{abstract}

Index Terms-Coupled limit-cycle oscillators, desynchronization, digital modified astrocyte model, field-programmable gate array (FPGA).

\section{INTRODUCTION}

A RTIFICIAL neural networks have been studied intensively during the past few years [1]-[12]. The motivations of these studies include the following: 1) exploitation of functional mechanisms of biological neural systems; 2) development of neural prosthetics (i.e., replacement of a biological neural system by an electronic circuit); and 3) development of artificial neural networks for use in engineering applications.

Astrocytes are part of the biological neural network that outnumber neurons by over a factor of five in the brain. They occur in the entire central nervous system (CNS) and perform many essential complex functions in the healthy CNS [13]. Moreover, they control the content of extracellular fluid and electrolyte homeostasis, regulate neurotransmitter release, and control synapse formation [14]. Recently, the importance of neuro-glial interactions for normal working of the CNS and peripheral nervous systems has been fully identified. Although astrocytes cannot generate action potentials, they respond to neuronal activities with an elevation of

Manuscript received June 20, 2013; revised December 14, 2013; accepted March 8, 2014. Date of publication March 31, 2014; date of current version December 16, 2014.

H. Soleimani and A. Ahmadi are with the Department of Electrical Engineering, Razi University, Kermanshah 67149-67346, Iran (e-mail: hsoleimani@razi.ac.ir; aahmadi@razi.ac.ir).

M. Bavandpour is with the Department of Electrical Engineering, Sharif University of Technology, Tehran 11365-11155, Iran (e-mail: bavandpour@ ee.sharif.ir).

D. Abbott is with the School of Electrical and Electronic Engineering, University of Adelaide, Adelaide, SA 5005, Australia (e-mail: derek.abbott@adelaide.edu.au).

Color versions of one or more of the figures in this paper are available online at http://ieeexplore.ieee.org.

Digital Object Identifier 10.1109/TNNLS.2014.2311839 their intracellular calcium levels. In this way, astrocytes can not only sense neuronal transmission, but also their calcium elevation that leads to the release of gliotransmitters, such as glutamate or adenosine triphosphate (ATP), which can regulate and control the synaptic strengths of neighboring neurons [15]-[17]. In other words, the amount of information transmitted across the synapse is modulated by astrocytic mechanisms.

Motivated by biological discoveries, a few computational astrocyte models have been developed to analyze the relationship between neurons and astrocytes. Nadkarni and Jung [18], [19] and Nadkarni et al. [20] proposed a dressed neuron model and provided a mathematical framework for the synaptic interactions between neurons and astrocytes, and a generalized dimensionless model for the tripartite synapse proposed in [21]. Recently, this model has been modified in order to be applied to a spatially extended neuron-astrocyte network [22]. This model considers the main aspects of neuron-astrocyte interactions. Therefore, this model will be useful for exploring the main types of astrocyte response and the resulting dynamical patterns that allow us to predict their changes with varying control parameters. Although previous theoretical astrocyte models can imitate most experimental measurements to a high degree of accuracy; due to their complexity, most of them are challenging to implement in large-scale artificial neural networks. Therefore, finding a new conveniently implementable astrocyte model is very important for use in the field of neural information coding memory and network dynamics.

Implementation of such neuronal models, targeting different platforms, has been reported in various studies. There exist three major approaches for this challenge.

1) Analog implementations are considered as a strong choice for direct implementation of bioinspired nervous systems [7]-[10]. In this approach, electronic components and circuits are utilized to mimic neurological dynamics. Due to its high performance and well-developed technology, an analog very large scale integration implementation enables prototyping of neuronal structures to test theories of bioinspired computation, structure, learning, and plasticity under real-time operation. This is of particular interest for sensory processing systems and biologically inspired robotics. Although these analog solutions are fast and efficient, they are inflexible and require a long development time.

2) Special purpose hardware has been developed to implement neurobiological functions using software-based 
systems for large-scale simulations, such as [11] and [12]. Even though these systems are flexible and biologically realistic with considerably high performance, the presented hardware approaches suffer from limited programmability and high cost. Unfortunately, the cost and development time make these approaches impractical for accessible general purpose large-scale simulations.

3) Recently, reconfigurable digital platforms have been used to realize nervous system models [1]-[6]. This approach uses digital computation to emulate individual neuronal behaviors in a parallel and distributed network architecture to implement a system-level dynamic. Although digital computation consumes more silicon area and power per function in comparison with analog counterparts, its development time is considerably lower and is not susceptible to power supply, thermal noise, or device mismatch. In addition, high-precision digital computation makes it possible to implement networks with high dynamic range, greater stability, reliability, and repeatability.

In this paper, a novel simple electronic circuit astrocyte model is proposed to exhibit the biological behaviors. This lays the groundwork for investigation of those types of neuronal synchronization that play an important role in biological information processing and diseases, such as Parkinson, essential tremor, and epilepsies [23]. The rest of this paper is organized as follows. Section II presents a brief background of the original model, while in Section III, the dynamic models of the proposed astrocyte model are explained. In Section IV, the synchrony of two coupled Hopf oscillators, and mathematical analysis of the role played by the proposed astrocyte model in neuronal activity are investigated. Digital implementation of the proposed single astrocyte model and the structure mentioned in the previous section are discussed in Section V. Section VI presents the results of implementation on scope to show the correctness of the theoretical deductions. Finally, Section VII concludes this paper.

\section{BACKGROUND}

Recently, a generalized dimensionless model [21] to reproduce the dynamics of intracellular $\mathrm{Ca}^{2+}$ waves produced by astrocytes has been proposed. This model has been modified for application to a spatially extended neuron-astrocyte network [22]. This model is useful for exploring the main types of astrocyte response and the resulting dynamical patterns that allow us to predict their changes with varying control parameters. These parameters will be introduced later in this section. This model is specified with the following set of equations:

$$
\begin{gathered}
\tau_{c} \frac{d c}{d t}=-c-c_{4}\left(c_{1} \frac{c^{2}}{1+c^{2}}-\frac{c_{e}^{2}}{1+c_{e}^{2}} \cdot \frac{c^{4}}{c^{4}+c_{2}^{4}}\right)+r+\beta \cdot S_{m} \\
f\left(c, c_{e}\right)=c_{1} \frac{c^{2}}{1+c^{2}}-\left(\frac{c_{e}^{2}}{1+c_{e}^{2}}\right)\left(\frac{c^{4}}{c^{4}+c_{2}^{4}}\right) \\
\tau_{c} \varepsilon_{c}=f\left(c, c_{e}\right)
\end{gathered}
$$

$$
\begin{gathered}
\tau_{G_{m}} \frac{d G_{m}}{d t}=\left(1+\tanh \left[S_{G_{m}}\left(c-h_{G_{m}}\right)\right]\right)\left(1-G_{m}\right)-\frac{G_{m}}{d_{G_{m}}} \\
\tau_{S_{m}} \frac{d S_{m}}{d t}=\left(1+\tanh \left[S_{S_{m}}\left(Z-h_{S_{m}}\right)\right]\right)\left(1-S_{m}\right)-\frac{S_{m}}{d_{S_{m}}}
\end{gathered}
$$

where $c$ is the calcium concentration in the astrocyte cytoplasm and $c_{e}$ denotes the calcium concentration within the endoplasmic reticulum. The parameters $\varepsilon_{c}$ and $\tau_{c}$ together define the characteristic time for calcium oscillations. The calcium influx from the extracellular space is sensitive to the production of secondary messenger $S_{m}$ (IP3), which is controlled by the factor $\beta$. The initial state of the calcium oscillation is controlled by the parameter $r$. The calcium exchange between the cytoplasm and the endoplasmic reticulum is defined by the nonlinear function $f\left(c, c_{e}\right)$. Increase of calcium concentration in the cytoplasm causes release of the astrocyte mediator $G_{m}$. The interaction between the astrocyte and neurons is controlled by the parameter $Z$ that affects the synaptic activity of the two coupled neurons.

\section{MODIFIED MODEL}

References [15]-[17] show that the variation of calcium is very important in the neuron-astrocyte interactions. Thus, we initiate to propose the model similar to [24] method, mainly, based on the dynamics of calcium concentration, and we omit ODE (4) that models the release of glutamate. Moreover, based on [22], $\tau_{S_{m}}>\tau_{c}$ and $\varepsilon_{c}$ in (1)-(5) is a small parameter, therefore $\tau_{S_{m}}>\tau_{c}>\varepsilon_{c} \tau_{c}$. This means that $c_{e}$ has fast dynamics in comparison with $c$ and $S_{m}$, and rapidly reaches its steady state (occurring when $d c_{e} / d t=0$, and thus $f\left(c, c_{e}\right)=$ $0)$. Therefore, $f\left(c, c_{e}\right)=0$ can be considered in (1)-(5). The reduced model is then

$$
\begin{aligned}
\tau_{c} \frac{d c}{d t} & =-c+r+\beta \cdot S_{m} \\
\tau_{S_{m}} \frac{d S_{m}}{d t} & =\left(1+\tanh \left[S_{S_{m}}\left(Z-h_{S_{m}}\right)\right]\right)\left(1-S_{m}\right)-\frac{S_{m}}{d_{S_{m}}} .
\end{aligned}
$$

Moreover, by time scaling $\tau=t / \tau_{S_{m}}$ and defining $c_{1}=$ $\tau_{S_{m}} / \tau_{c}, c_{2}=\tau_{S_{m}} r / \tau_{c}, c_{3}=\tau_{S_{m}} \beta / \tau_{c}, c_{4}=S_{S_{m}}, c_{5}=h_{S_{m}}$, $c_{6}=1 / d_{S_{m}}$, and renaming $x=c, y=S_{m}$, the structure of the model is simplified from (1)-(3) and (5) to (6) and (7). On the other hand, for implementation of tanh, a programmable tanhlike function is considered [25] as follows:

$$
\tanh (x)=\operatorname{sgn}(x)\left[1+\frac{1}{2^{\left\lfloor 2^{n}|x|\right\rfloor}}\left(\frac{2^{n}|x|-\left\lfloor 2^{n}|x|\right\rfloor}{2}-1\right)\right]
$$

where $n$ is an integer number and determines the steepness of the function. This approximation uses an exp-like $\left(2^{x}\right)$ function instead of $e^{x}$ that is given by

$$
2^{x} \cong 2^{\lfloor x\rfloor}[1+(x-\lfloor x\rfloor)]
$$

where $\lfloor x\rfloor$ represents the integer part of $x$, and $x-\lfloor x\rfloor$ indicates the fractional part of $x$. Using this approximation, several sigmoid-like functions can be designed. Finally, by replacement of (8) in (7), a digital implementation of the 


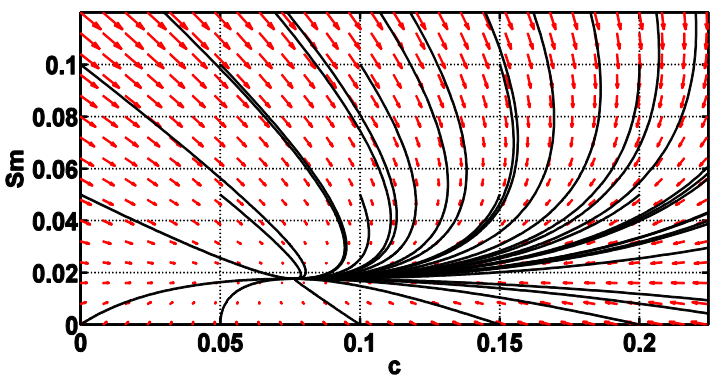

(a)

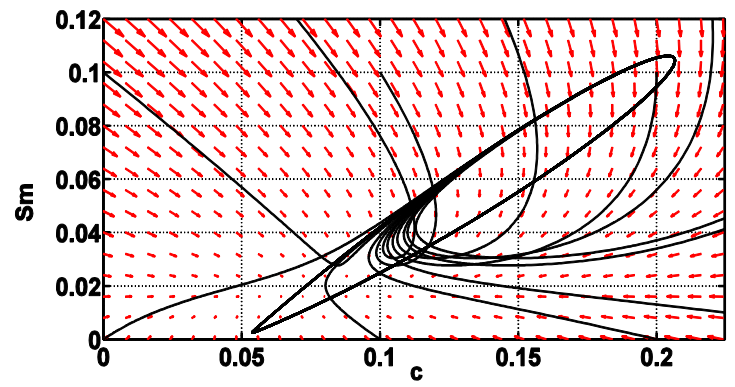

(c)

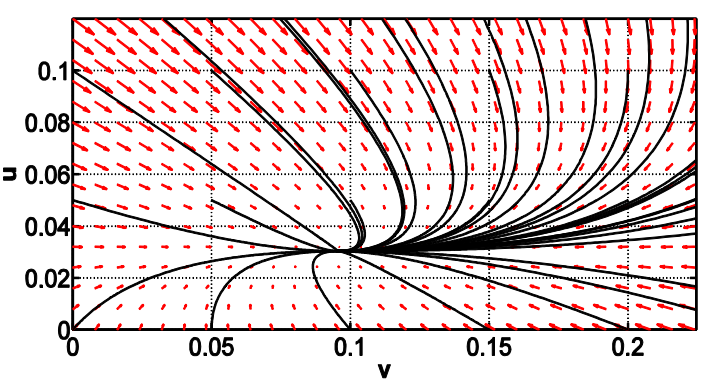

(b)

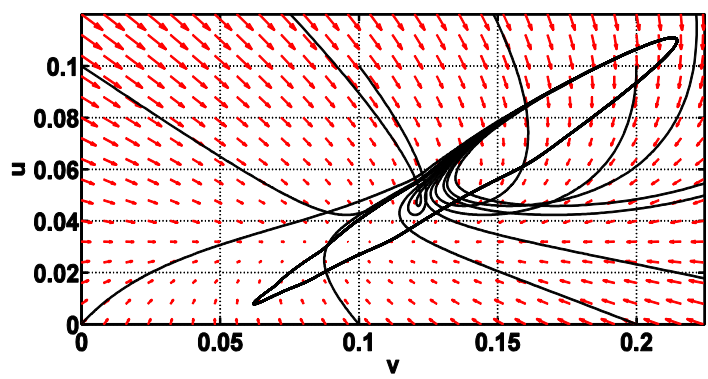

(d)

Fig. 1. Nonautonomous behaviors in the phase portraits for both proposed and original models. (a) Behavior of the original model when input is equal to zero. (b) Behavior of the proposed model when input is equal to zero. (c) Behavior of the original model when input is equal to sin $(0.15 t)$. (d) Behavior of the proposed model when input is equal to $\sin (0.15 t)$.

TABLE I

Astrocyte Parameter VAlues

\begin{tabular}{cccc}
\hline \hline Parameters & Value & Parameters & Value \\
\hline$c_{1}$ & 1 & $c_{4}$ & 1 \\
$c_{2}$ & 0.05 & $c_{5}$ & 2 \\
$c_{3}$ & 1.5 & $c_{6}$ & 2 \\
\hline
\end{tabular}

modified model can be formed according to the following:

$$
\begin{aligned}
\dot{v}= & -c_{1} v+c_{2}+c_{3} u \\
\dot{u}= & \left(1+\operatorname{sgn}\left(\left[c_{4}\left(Z-c_{5}\right)\right]\right)\left[1+\frac{1}{2^{\left[2^{n}\left|c_{4}\left(Z-c_{5}\right)\right|\right\rfloor}}\right.\right. \\
& \left.\left.\times\left(\frac{2^{n}\left|c_{4}\left(Z-c_{5}\right)\right|-\left\lfloor 2^{n}\left|c_{4}\left(Z-c_{5}\right)\right|\right\rfloor}{2}-1\right)\right]\right)(1-u)-c_{6} u
\end{aligned}
$$

where $\dot{v}=d x / d \tau$ and $\dot{u}=d y / d \tau$. We set the control parameters $c_{1}, c_{2}, c_{3}, c_{4}, c_{5}$, and $c_{6}$ to the values listed in Table I that are taken from [24].

The nullclines of the proposed astrocyte model provide insights into the similarity in dynamics in comparison with the original model. For obtaining the nullclines and equilibrium points of a dynamical system in the steady state, the inputs and derivatives of the state variables are set to zero. So, considering $\dot{v}=0$ and $\dot{u}=0$, the nullclines are given by

$$
\begin{gathered}
-c_{1} v+c_{2}+c_{3} u=0 \\
\left(1+\operatorname{sgn}\left(\left[c_{4}\left(Z-c_{5}\right)\right]\right)\left[1+\frac{1}{2^{\left\lfloor 2^{n}\left|c_{4}\left(Z-c_{5}\right)\right|\right\rfloor}}\right.\right. \\
\left.\left.\times\left(\frac{2^{n}\left|c_{4}\left(Z-c_{5}\right)\right|-\left\lfloor 2^{n}\left|c_{4}\left(Z-c_{5}\right)\right|\right\rfloor}{2}-1\right)\right]\right) \\
(1-u)-c_{6} u=0
\end{gathered}
$$

where $Z=0$, there is one fixed point with the coordinate $\left(v_{\mathrm{eq}}, u_{\mathrm{eq}}\right)=(0.095,0.03)$. To determine the type of this equilibrium point, eigenvalues of the system are calculated as follows:

$$
J(v, u)=J\left(v_{\mathrm{eq}}, u_{\mathrm{eq}}\right)=\left[\begin{array}{cc}
-1 & 1.5 \\
0 & -2.0625
\end{array}\right]
$$

and eigenvalue equation is

$$
P(\lambda)=\lambda^{2}+3.0625 \lambda+2.0625
$$

and by solving this equation, we find two real eigenvalues equal to $\left(\lambda_{1}, \lambda_{1}\right)=(-2.0625,-1)$. This leads to the occurrence of a sink point at $(0.095,0.03)$, which attracts any point in the phase portrait. So, the behavior of the proposed model without input is quite similar to the original model that is shown in Fig. 1(a) and (b).

When the input $Z$ is applied to the model, the equilibrium points are

$$
\begin{aligned}
& v_{\text {eq }}=\left(c_{2}+c_{3} u_{\text {eq }}\right) / c_{1} \\
& u_{\text {eq }}=l /\left(l+c_{6}\right)
\end{aligned}
$$

where

$$
\begin{aligned}
l=\left(1+\operatorname{sgn}\left(\left[c_{4}\left(Z-c_{5}\right)\right]\right)\left[1+\frac{1}{2^{\left\lfloor 2^{n}\left|c_{4}\left(Z-c_{5}\right)\right|\right\rfloor}}\right.\right. \\
\left.\left.\quad \times\left(\frac{2^{n}\left|c_{4}\left(Z-c_{5}\right)\right|-\left\lfloor 2^{n}\left|c_{4}\left(Z-c_{5}\right)\right|\right\rfloor}{2}-1\right)\right]\right)
\end{aligned}
$$

and it is clear that $0<l<2$. By shifting $\bar{v}=v-v_{\mathrm{eq}}$ and $\bar{u}=u-u_{\mathrm{eq}}$, the equilibrium point is shifted to $(0,0)$. In the new coordinates, the proposed model is represented by

$$
\begin{aligned}
\dot{\bar{v}} & =-c_{1} \bar{v}+c_{3} \bar{u} \\
\dot{\bar{u}} & =-\left(l+c_{6}\right) \bar{u}
\end{aligned}
$$


considering that the Lyapunov function $V_{1}=1 / 2 \bar{u}^{2}$ leads to $\dot{V}_{1}=-\left(l+c_{6}\right) \bar{u}^{2}<c_{6} \bar{u}^{2}$. So, $\bar{u}$ is globally exponentially stable. Next, based on the following useful lemma and since the unforced term of $\bar{v}$ (i.e., $-c_{1} \bar{v}$ ) is globally exponentially stable, it is input-to-state stable with $\bar{u}$.

Lemma 1 [26]: $f(t, x, u)$ is continuously differentiable and globally Lipschitz in $(x, u)$, uniformly in $t$, if the unforced system $\dot{x}=f(t, x, 0)$ has a globally exponentially stable equilibrium point at the origin $x=0$, then the system $\dot{x}=f(t, x, u)$ is input-to-state stable.

Proof [26]: Consider the system $\dot{x}=f(t, x, u)$ as a perturbation of the unforced system $\dot{x}=f(t, x, 0)$. (The converse Lyapunov) Theorem 1 shows that the unforced system $\dot{x}=f(t, x, 0)$ has a Lyapunov function $V(t, x)$ that satisfies the inequality of the theorem globally. Due to the uniform global Lipschitz property of $f$, the perturbation term satisfies $\|f(t, x, u)-f(t, x, 0)\| \leq L\|u\|$ for all $t \geq t_{0}$ and all $(x, u)$. The derivative of $V$ with respect to $\dot{x}=f(t, x, u)$ satisfies

$$
\begin{aligned}
\dot{V} & =\frac{\partial V}{\partial t}+\frac{\partial V}{\partial x} f(t, x, 0)+\frac{\partial V}{\partial x}[f(t, x, u)-f(t, x, 0)] \\
& \leq-b_{3}\|x\|^{2}+b_{4}\|x\| L\|u\| .
\end{aligned}
$$

To use the term $-b_{3}\|x\|^{2}$ to dominate $b_{4} L\|x\|\|\mid\| u \|$ for large $\|x\|$, we rewrite the foregoing inequality as

$$
\dot{V} \leq-b_{3}(1-\theta)\|x\|^{2}-b_{3} \theta\|x\|^{2}+b_{4} L\|x\|\|\mid u\|
$$

where $0<\theta<1$. Then

$$
\dot{V} \leq-b_{3}(1-\theta)\|x\|^{2} \quad \forall\|x\| \geq \frac{b_{4} L\|u\|}{b_{3} \theta}
$$

for all $(t, x, u)$. Hence, the conditions of Theorem 3 are satisfied with $\alpha_{1}(r)=b_{1} r^{2}, \alpha_{2}(r)=b_{2} r^{2}$, and $\rho(r)=$ $\left(b_{4} L / b_{3} \theta\right) r$, and we conclude that the system is input-to-state stable with $\gamma(r)=\sqrt{b_{2} / b_{1}}\left(b_{4} L / b_{3} \theta\right) r$. Note that Lemma 1 requires a globally Lipschitz function $f$ and global exponential stability of the origin of the unforced system to give input-tostate stability.

Therefore, using Lemma 2 in the following, it can be observed that the origin is a globally asymptotically equilibrium point of (19) and (20). Thus, for all values of $c_{i}$, the proposed model is globally asymptotically stable. So, similar to the original model, there is also a stable limit cycle in the proposed model that is shown in Fig. 1(c) and (d). It is observed that, in both nonautonomous systems, the phase portraits ultimately converge to the approximately same limit cycle.

Lemma 2 [26]: Under the stated assumptions, if the system $\dot{x_{1}}=f_{1}\left(t, x_{1}, x_{2}\right)$, with $x_{2}$ as input, is input-to-state stable and the origin of $\dot{x_{2}}=f_{2}\left(t, x_{2}\right)$ is globally uniformly asymptotically stable, then the origin of the cascade system $\dot{x_{1}}=f_{1}\left(t, x_{1}, x_{2}\right)$ and $\dot{x_{2}}=f_{2}\left(t, x_{2}\right)$ is globally uniformly asymptotically stable.

Proof [26]: Let $t_{0} \geq 0$ be the initial time. The solutions of $\dot{x_{1}}=f_{1}\left(t, x_{1}, x_{2}\right)$ and $\dot{x_{2}}=f_{2}\left(t, x_{2}\right)$ satisfy

$$
\begin{aligned}
\left\|x_{1}(t)\right\| & \leq \beta_{1}\left(\left\|x_{1}(s)\right\|, t-s\right)+\gamma_{1}\left(\sup _{s \leq \tau \leq t}\left\|x_{2}(\tau)\right\|\right) \\
\left\|x_{2}(t)\right\| & \leq \beta_{2}\left(\left\|x_{2}(s)\right\|, t-s\right)
\end{aligned}
$$

globally, where $t \geq s \geq t_{0}, \beta_{1}$ and $\beta_{2}$ are class $\kappa \varrho$ functions, and $\gamma_{1}$ is a class of $\kappa$ function. Apply (24) with $s=\left(t+t_{0}\right) / 2$ to obtain

$$
\left\|x_{1}(t)\right\| \leq \beta_{1}\left(\left\|x_{1}\left(\frac{t+t_{0}}{2}\right)\right\|, \frac{t-t_{0}}{2}\right)+\gamma_{1}\left(\sup _{\frac{t+t_{0}}{2} \leq \tau \leq t}\left\|x_{2}(\tau)\right\|\right) .
$$

To estimate $x_{1}\left(\left(t+t_{0}\right) / 2\right)$, apply (24) with $s=t_{0}$ and $t$ replaced by $\left(t+t_{0}\right) / 2$ to obtain

$$
\left\|x_{1}\left(\frac{t+t_{0}}{2}\right)\right\| \leq \beta_{1}\left(\left\|x_{1}\left(t_{0}\right)\right\|, \frac{t-t_{0}}{2}\right)+\gamma_{1}\left(\sup _{t_{0} \leq \tau \leq \frac{t+t_{0}}{2}}\left\|x_{2}(\tau)\right\|\right)
$$

Using (25), we obtain

$$
\begin{aligned}
& \sup _{t_{0} \leq \tau \leq \frac{t+t_{0}}{2}}\left\|x_{2}(\tau)\right\| \leq \beta_{2}\left(\left\|x_{2}\left(t_{0}\right)\right\|, 0\right) \\
& \sup _{\frac{t+t_{0}}{2} \leq \tau \leq t}\left\|x_{2}(\tau)\right\| \leq \beta_{2}\left(\left\|x_{2}\left(t_{0}\right)\right\|, \frac{t-t_{0}}{2}\right) .
\end{aligned}
$$

Substituting (27)-(29) into (26) and using the inequalities

$$
\begin{aligned}
\left\|x_{1}\left(t_{0}\right)\right\| & \leq\left\|x\left(t_{0}\right)\right\|, \quad\left\|x_{2}\left(t_{0}\right)\right\| \leq\left\|x\left(t_{0}\right)\right\| \\
\left\|x_{(}(t)\right\| & \leq\left\|x_{1}(t)\right\|+\left\|x_{2}(t)\right\|
\end{aligned}
$$

yield

$$
\|x(t)\| \leq \beta\left(\left\|x\left(t_{0}\right)\right\|, t-t_{0}\right)
$$

where

$$
\begin{aligned}
\beta(r, s)= & \beta_{1}\left(\beta_{1}\left(r, \frac{s}{2}\right)+\gamma_{1}\left(\beta_{2}(r, 0)\right), \frac{s}{2}\right) \\
& +\gamma_{1}\left(\beta_{2}\left(r, \frac{s}{2}\right)\right)+\beta_{2}(r, s) .
\end{aligned}
$$

It can be easily verified that $\beta$ is a class $\kappa \varrho$ function for all $r \geq 0$. Hence, the origin of $\dot{x_{1}}=f_{1}\left(t, x_{1}, x_{2}\right)$ and $\dot{x_{2}}=$ $f_{2}\left(t, x_{2}\right)$ is globally uniformly asymptotically stable.

\section{Application to Astrocyte Interactions With Two COUPLED Hopf OSCILlATORS}

For the investigation of proposed astrocyte model behaviors in a neuronal network, we apply it to a particular example. In this section, we consider two coupled oscillators modeled by the Hopf system as follows [27]:

$$
\begin{aligned}
& \dot{x}=\left(1-x^{2}-y^{2}\right) x+\omega_{i} y+x+I \\
& \dot{y}=\left(1-x^{2}-y^{2}\right) y-\omega_{i} x+y .
\end{aligned}
$$

Oscillators states $\left(x_{i}, y_{i}\right)$ asymptotically converge to the stable circular limit cycle with radius one. They oscillate with the frequencies $\omega_{i}, i=1,2, \omega_{1} \neq \omega_{2}$. Suppose oscillators are coupled linearly with the coupling strength $G>0$ as follows:

$$
\begin{aligned}
& \dot{x}=\left(1-x^{2}-y^{2}\right) x+\omega_{i} y+x+G\left(x_{k}-x_{i}\right) \\
& \dot{y}=\left(1-x^{2}-y^{2}\right) y-\omega_{i} x+y+G\left(y_{k}-y_{i}\right) .
\end{aligned}
$$




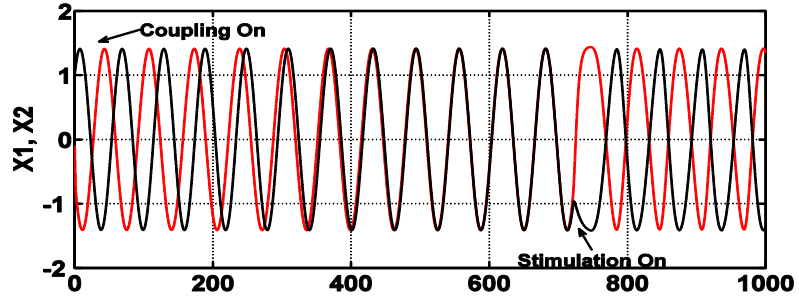

(a)

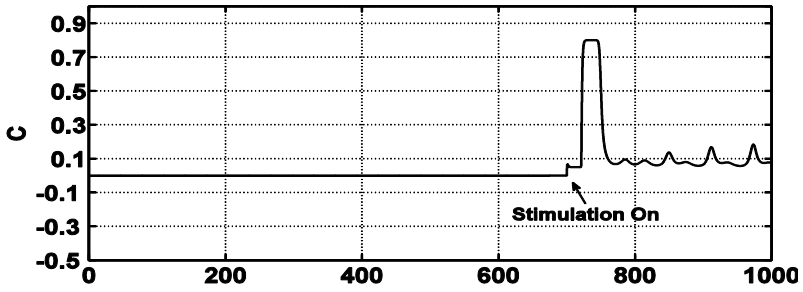

(c)

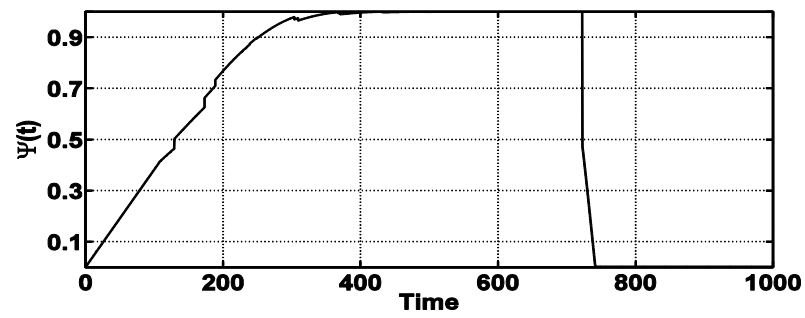

(e)

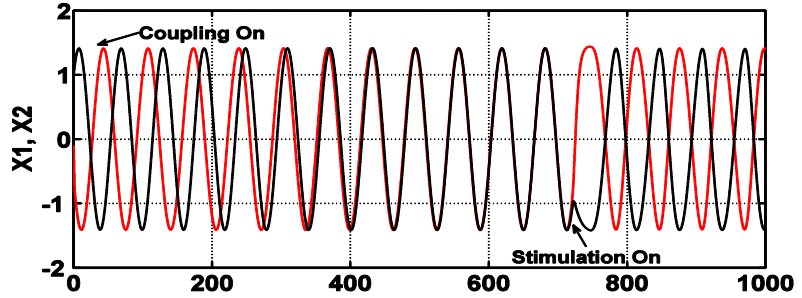

(b)

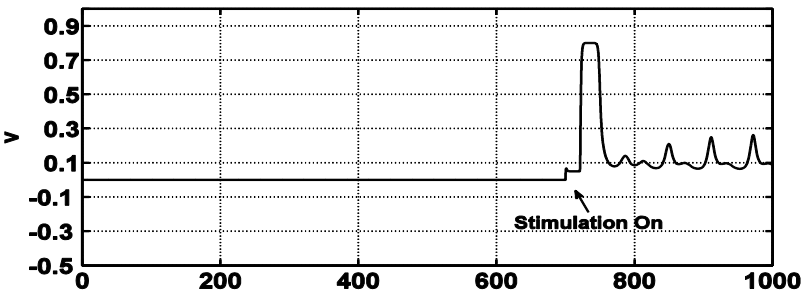

(d)

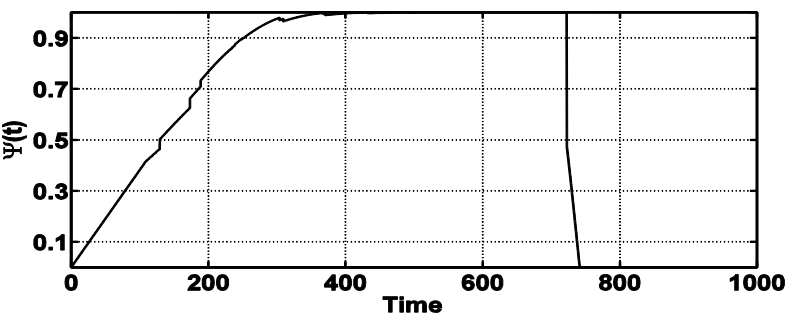

(f)

Fig. 2. Effect of varying coupling strength $G$ for the original and proposed models. (a) $x_{1}, x_{2}$ for two coupled oscillators with regulating by original astrocyte model. (b) $x_{1}, x_{2}$ for two coupled oscillators with regulating by proposed astrocyte model. (c) $c$ (the output of original astrocyte model). (d) $v$ (the output of proposed astrocyte model). (e) Synchronization index for the original astrocyte model. (f) Synchronization index for the proposed astrocyte model.

In the polar coordinates, $\left(r_{i} e^{j \theta_{i}}=x_{i}+j y_{i}\right)$ can be represented as

$$
\begin{aligned}
& \dot{r_{i}}=r_{i}\left(1-G-r_{i}^{2}\right)+G r_{k} \cos \left(\theta_{k}-\theta_{i}\right) \\
& \dot{\theta_{i}}=\omega_{i}+G r_{k} / r_{i} \sin \left(\theta_{k}-\theta_{i}\right) \\
& \quad i, k \in\{1,2\} \quad \text { and } \quad i \neq k .
\end{aligned}
$$

To clarify the results, we define the concept of synchronization. If $x_{1}(t)=x_{2}(t)$ for all $t$, perfect synchronization occurs. For asymmetric initial conditions, that is $x_{1}(0)=x_{2}(0)$, the equality holds asymptotically. For asymmetric systems with $I_{1}=I_{2}$, perfect synchrony is not expected. A solution $\left(x_{1}(t), x_{2}(t)\right)$ is synchronized if $x_{1}(t)$ and $x_{2}(t)$ remain close to each other that is $\left|x_{1}(t)-x_{2}(t)\right| \leq s(t) \varepsilon\left|I_{1}-I_{2}\right|$, where $s(t) \geq 0$ is a continuous function with $\lim _{t \rightarrow+\infty} s(t)=0$ and $\varepsilon$ is a constant. Moreover, we define desynchronization as the case where $\lim _{t \rightarrow+\infty}\left|\theta_{1}(t)-\theta_{2}(t)\right|=\pi$. To quantify the synchronization concept, the following complex order parameter is defined [28]:

$$
\Psi(t) e^{j \Omega(t)}=\left(\frac{1}{N}\right) \sum_{i=1}^{N} e^{j \theta_{i}(t)}
$$

where $\Psi(t)$ is the synchronization index, $\Omega(t)$ is the mean phase, and $N$ is the number of oscillators (here $N=2$ ). It is clear that $0 \leq \Psi \leq 1$ and

$$
\theta(t)=2 \pi \frac{t-t_{k}}{t_{k+1}-t_{k}}
$$

where $t \in\left[t_{k}, t_{k+1}\right]$, and $t_{k}$ is the onset time of the $k$ th pick of $i$ th oscillator signal. For two oscillators, we have

$$
|\Psi(t)|=\frac{1}{2}\left|e^{j \theta_{1}(t)}+e^{j \theta_{2}(t)}\right|
$$

then

$|\Psi(t)|=\frac{1}{2} \sqrt{2+2 \cos \theta_{1}(t) \cdot \cos \theta_{2}(t)+\sin \theta_{1}(t) \cdot \sin \theta_{2}(t)}$

where $\Psi(t)=1$ corresponds to coincidence of phases, and desynchronization is characterized by $\Psi(t)=0$. Finally, the scheme of the neural network is described as

$$
\begin{aligned}
\dot{x_{1}} & =\left(1-x_{1}^{2}-y_{1}^{2}\right) x_{1}+\omega_{1} y_{1}+x_{1}+G\left(x_{2}-x_{1}\right)+\rho_{1} v \\
\dot{y_{1}} & =\left(1-x_{1}^{2}-y_{1}^{2}\right) y_{1}+\omega_{1} x_{1}+y_{1}+G\left(y_{2}-y_{1}\right)+\rho_{1} v \\
\dot{x_{2}} & =\left(1-x_{2}^{2}-y_{2}^{2}\right) x_{2}+\omega_{2} y_{2}+x_{2}+G\left(x_{1}-x_{2}\right)+\rho_{2} v \\
\dot{y_{2}} & =\left(1-x_{2}^{2}-y_{2}^{2}\right) y_{2}+\omega_{2} x_{2}+y_{2}+G\left(y_{1}-y_{2}\right)+\rho_{2} v \\
\dot{v} & =-c_{1}+c_{2}+c_{3} u \\
\dot{u} & =\left(1+\operatorname{sgn}\left(\left[c_{4}\left(Z-c_{5}\right)\right]\right)\left[1+\frac{1}{2^{\left[2^{n}\left|c_{4}\left(Z-c_{5}\right)\right|\right\rfloor}}\right.\right. \\
& \left.\left.\times\left(\frac{2^{n}\left|c_{4}\left(Z-c_{5}\right)\right|-\left\lfloor 2^{n}\left|c_{4}\left(Z-c_{5}\right)\right|\right\rfloor}{2}-1\right)\right]\right)(1-u)-c_{6} u .
\end{aligned}
$$




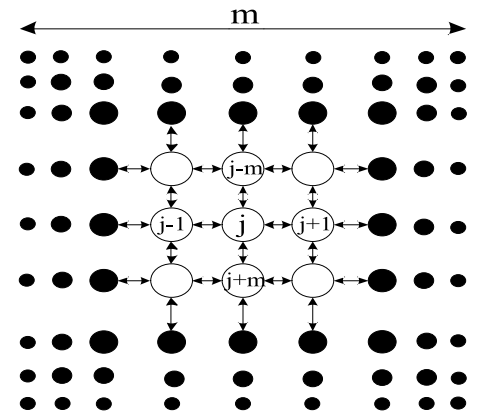

(a)

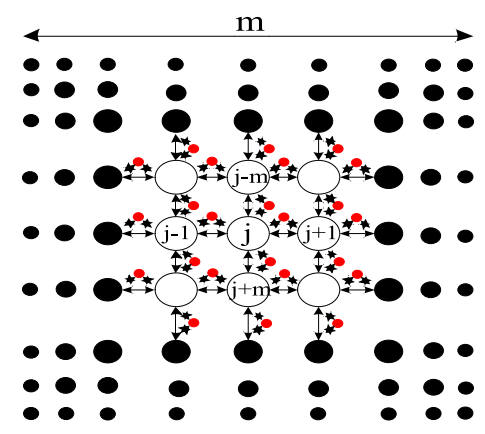

(c)

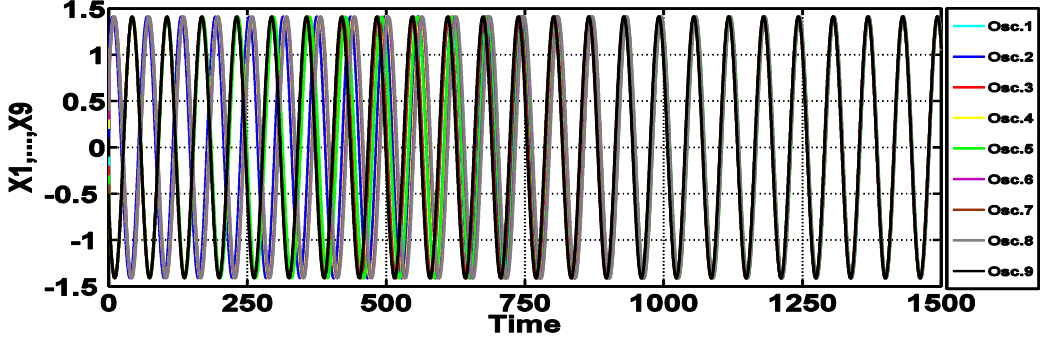

(b)

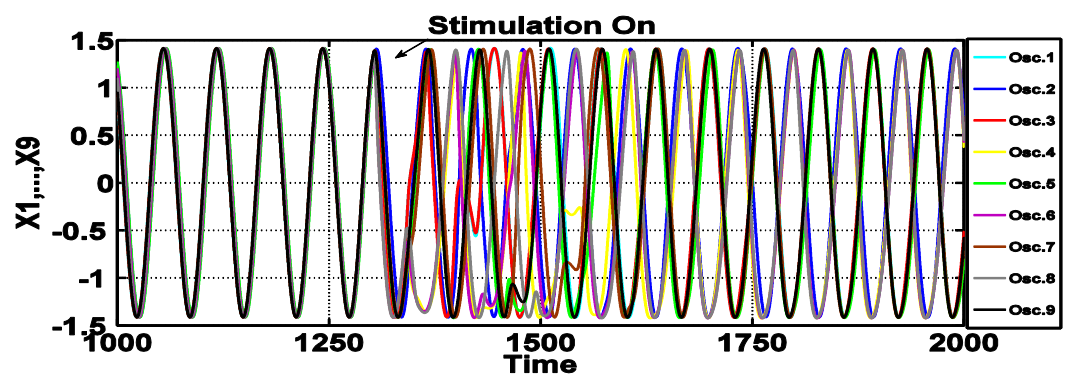

(d)

Fig. 3. (a) Four von Neumann neighborhood network with four neighborhood oscillator connections. (b) $x_{1}$ to $x_{9}$ for a $3 \times 3$ matrix of oscillators that have been coupled based on four von Neumann neighborhood. (c) Four von Neumann neighborhood network with four neighborhood oscillator connections and proposed astrocyte between each two oscillators. (d) $x_{1}-x_{9}$ for a $3 \times 3$ matrix of oscillators and four astrocytes with a von Neumann neighborhood.

The input $Z=a\left(x_{1}+y_{1}+x_{2}+y_{2}\right)$ for the proposed model triggers the $y$-dynamics, then $y$ affects the $x$ dynamics through the term $c_{3} y$. Since $v$ has a crucial role in (10) and (11) [c in (1)-(5), respectively], we consider $v$ as the model output. Finally, $v$ controls (desynchronizes) the two coupled oscillators through the stimulation terms $\rho_{1} x$ and $\rho_{1} y$. Note that, among gliotransmitters, which astrocytes release, glutamate has an excitatory effect, whereas ATP has an inhibitory effect. This fact is modeled by considering a positive sign for excitation and negative sign for inhibition. For the simulations shown in Fig. 2, $G=0.005, \rho_{1}=0.14, \rho_{2}=-0.18, \omega_{1}=\omega_{2}=$ $0.1, a=8, c_{1}=1, c_{2}=0.05, c_{3}=1.5, c_{4}=1, c_{5}=2$, and $c_{6}=2$.

\section{Network Behaviors}

In this section, to investigate the performance of proposed astrocyte model, two well-known network structures are presented. In these structures, oscillators are coupled with other neighborhood oscillators through a weak coupling strength. Moreover, each two connected oscillators on the surface interact with an astrocyte. In these cases, the behaviors of each oscillator and astrocyte, which lead to synchronization and desynchronization of the network, are investigated. The main purpose behind this investigation is the fact that the proposed astrocyte model can optimally increase the input current of the individual oscillators to prevent synchronization in the neural networks. In the networks, depending on the initial values of oscillators and number of connections, different synchrony and asynchrony times occur. Due to the interaction between oscillators and astrocytes, synchrony of two neighbor oscillators is halted and convergence is attempted asynchronously.

\section{A. Four Von Neumann Neighborhood in Flat Form}

The first structure is a flat network with von Neumann neighborhood structure [29] that is shown in Fig. 3(a). In this structure, each oscillator is connected to four other neighbors located up, down, left, and right. To create weak coupling between oscillators, we utilize the $x$ and $y$ state variables of the neighbors. For the $j$ th oscillator, we have

$$
\begin{aligned}
\dot{x_{j}}= & \left(1-x_{j}^{2}-y_{j}^{2}\right) x_{j}+\omega_{j} y_{j}+x_{j}+G\left(\left(x_{j-1}-x_{j}\right)+\left(x_{j+1}-x_{j}\right)\right. \\
& \left.+\left(x_{j-m}-x_{j}\right)+\left(x_{j+m}-x_{j}\right)\right) \\
\dot{y_{j}}= & \left(1-x_{j}^{2}-y_{j}^{2}\right) y_{j}-\omega_{j} x_{j}+y_{j}+G\left(\left(y_{j-1}-y_{j}\right)+\left(y_{j+1}-y_{j}\right)\right. \\
& \left.+\left(y_{j-m}-y_{j}\right)+\left(y_{j+m}-y_{j}\right)\right)
\end{aligned}
$$

where $m$ is the number of oscillators in each row. Note that, due to the flat form of this structure, the oscillators on the edges are in contact with just two or three neighbors. For example, the oscillator that is in the upper right hand corner connects with two down and left neighbors.

To clarify the effect of connections in the network, we give the network different initial values for $x$ and $y$ and $G=0.001$. The behavior of this network is shown in Fig. 3(b). We see that all oscillators converge to a common signal with the same phase and frequency. This phenomenon leads to synchrony. To prevent this phenomenon, an astrocyte cell is added between each of the two connected oscillators on the surface. The scheme of the neural network is shown in 


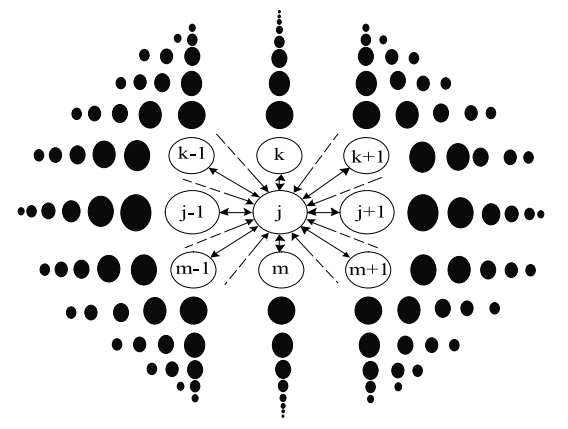

(a)

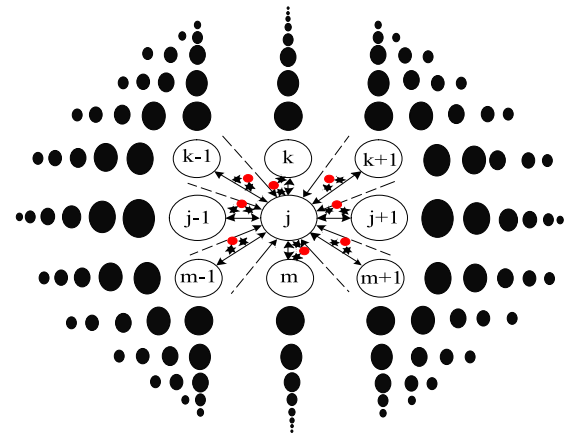

(c)

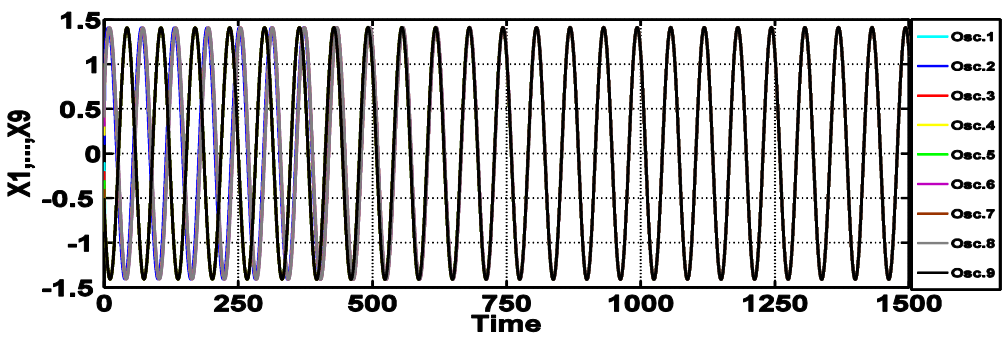

(b)

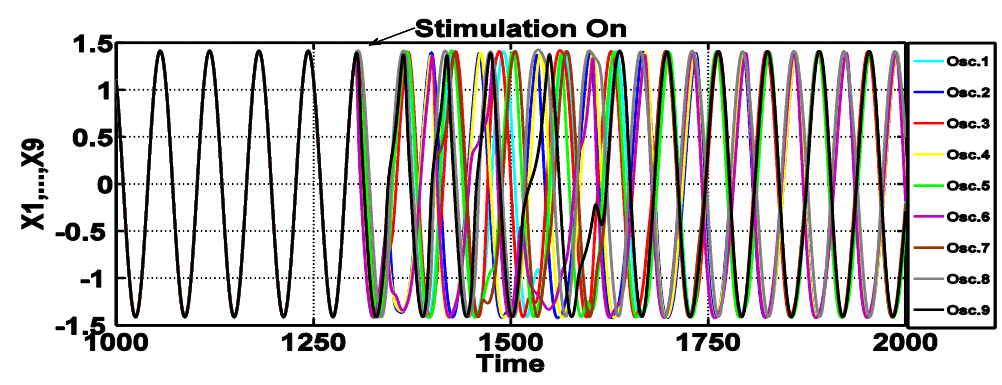

(d)

Fig. 4. (a) Full connection networked oscillators. (b) Network with four neighborhood oscillator connections. (b) $x_{1}-x_{9}$ for a $3 \times 3$ matrix of oscillators, which have been fully connected. (c) Full connection networked oscillators and proposed astrocyte between each two oscillators. (d) $x_{1}-x_{9}$ for a $3 \times 3$ matrix of fully connected oscillators with astrocytes.

Fig. 3(c) and described as

$$
\begin{aligned}
& \dot{x_{j}}=\left(1-x_{j}^{2}-y_{j}^{2}\right) x_{j}+\omega_{j} y_{j}+x_{j}+G\left(\left(x_{j-1}-x_{j}\right)+\left(x_{j+1}-x_{j}\right)\right. \\
& \left.+\left(x_{j-m}-x_{j}\right)+\left(x_{j+m}-x_{j}\right)\right)+\rho_{1}\left(v_{l}+v_{l+1}\right) \\
& +\rho_{2}\left(v_{l+2}+v_{l+3}\right) \\
& \dot{y}_{j}=\left(1-x_{j}^{2}-y_{j}^{2}\right) y_{j}-\omega_{j} x_{j}+y_{j}+G\left(\left(y_{j-1}-y_{j}\right)+\left(y_{j+1}-y_{j}\right)\right. \\
& \left.+\left(y_{j-m}-y_{j}\right)+\left(y_{j+m}-y_{j}\right)\right)+\rho_{1}\left(v_{l}+v_{l+1}\right) \\
& +\rho_{2}\left(v_{l+2}+v_{l+3}\right) \\
& \dot{v_{l}}=-c_{1}+c_{2}+c_{3} u_{l} \\
& \dot{u}_{l}=\left(1+\operatorname{sgn}\left(\left[c_{4}\left(Z_{l}-c_{5}\right)\right]\right)\left[1+\frac{1}{2^{\left\lfloor 2^{n}\left|c_{4}\left(Z_{l}-c_{5}\right)\right|\right.}}\right.\right. \\
& \left.\left.\times\left(\frac{2^{n}\left|c_{4}\left(Z_{l}-c_{5}\right)\right|-\left\lfloor 2^{n}\left|c_{4}\left(Z_{l}-c_{5}\right)\right|\right\rfloor}{2}-1\right)\right]\right) \\
& \times\left(1-u_{l}\right)-c_{6} u_{l}
\end{aligned}
$$

where $Z_{l}$ is equal to sum of $x$ and $y$ variable states of two connected oscillators by multiplying a constant coefficient, $a$, and $v_{l}, v_{l+1}, v_{l+2}$, and $v_{l+3}$ are outputs of all possible connected astrocytes to an oscillator in the network. The simulation results with $\rho_{1}=0.12$ and $\rho_{2}=-0.22$ are shown in Fig. 3(d). As it is expected, the proposed astrocyte model modifies the oscillators' inputs in the network by providing appropriate feedback actions. Therefore, runaway excitation is compensated and normal asynchronous behavior is again resumed quickly.

\section{B. Full Connection Networked Oscillators}

In this network, we consider a more realistic situation with fully connected networked oscillators to survey the role of
TABLE II

SUMMARIZED COMPARISON BETWEEN TWO NETWORKS

\begin{tabular}{ccc}
\hline \hline & 4 Von Neumann Neighborhood & Full Connection \\
\hline Synchrony Time(s) & 750 & 500 \\
Asynchrony Time(s) & 1600 & 1700 \\
Stimulation Time(s) & 1300 & 1300 \\
No. of Connections & 12 & 36 \\
\hline
\end{tabular}

astrocyte in prevention of synchrony. The fully connected networked oscillators are shown in Fig. 4(a). The number of connections in this structure is much greater than the previous network that contributes to the occurrence of a shorter synchrony time. Moreover, the fully connected network is much more resistant against desynchronization. In this structure, all oscillators are fully connected to each other, utilizing the $x$ and $y$ state variables of the neighbors for the $j$ th oscillator as follows:

$$
\begin{aligned}
& \dot{x_{j}}=\left(1-x_{j}^{2}-y_{j}^{2}\right) x_{j}+\omega_{j} y_{j}+x_{j}+G \sum_{p=1}^{h}\left(x_{p}-x_{j}\right) \\
& \dot{y_{j}}=\left(1-x_{j}^{2}-y_{j}^{2}\right) y_{j}-\omega_{j} x_{j}+y_{j}+G \sum_{p=1}^{h}\left(y_{p}-y_{j}\right)
\end{aligned}
$$

where $h$ is the number of oscillators in the network. To clarify the effect of these connections in the network, we give the network different initial values for $x$ and $y$ and $G=0.001$. The behavior of this network is shown in Fig. 4(b). We see that all oscillators attain synchrony state in a short time in comparison with the previous network. Like the previous 




(c)

Fig. 5. Arithmetic structure. (a) Neuronal network structure. (b) Coupled Hopf structure. (c) Modified astrocyte model.

section, to prevent this phenomenon, an astrocyte cell is added between each two connected oscillators. The scheme of the neural network is shown in Fig. 4(c) and described as

$$
\begin{aligned}
& \dot{x_{j}}=\left(1-x_{j}^{2}-y_{j}^{2}\right) x_{j}+\omega_{j} y_{j}+x_{j}+G \sum_{p=1}^{h}\left(x_{p}-x_{j}\right) \\
& +\rho_{1}\left(v_{l}+v_{l+1}\right)+\rho_{2}\left(v_{l+2}+v_{l+3}\right) \\
& \dot{y_{j}}=\left(1-x_{j}^{2}-y_{j}^{2}\right) y_{j}-\omega_{j} x_{j}+y_{j}+G \sum_{p=1}^{h}\left(y_{p}-y_{j}\right) \\
& +\rho_{1}\left(v_{l}+v_{l+1}\right)+\rho_{2}\left(v_{l+2}+v_{l+3}\right) \\
& \dot{v_{l}}=-c_{1}+c_{2}+c_{3} u_{l} \\
& \dot{u}_{l}=\left(1+\operatorname{sgn}\left(\left[c_{4}\left(Z_{l}-c_{5}\right)\right]\right)\left[1+\frac{1}{2^{\left\lfloor 2^{n}\left|c_{4}\left(Z_{l}-c_{5}\right)\right|\right]}}\right.\right. \\
& \left.\left.\times\left(\frac{2^{n}\left|c_{4}\left(Z_{l}-c_{5}\right)\right|-\left\lfloor 2^{n}\left|c_{4}\left(Z_{l}-c_{5}\right)\right|\right\rfloor}{2}-1\right)\right]\right) \\
& \times\left(1-u_{l}\right)-c_{6} u_{l} \text {. }
\end{aligned}
$$

The simulation results with the same parameters and initial conditions to previous network are shown in Fig. 4(d). In accordance with the previous work, the proposed astrocyte modifies the oscillator inputs by providing appropriate feedback actions. Therefore, stimulation is compensated and normal asynchronous behavior is resumed. This process is done after a longer time in comparison with the previous network. As far as simulations show, by increasing the number of connections for each of the oscillator in a network, synchrony and asynchrony times are changed. A summarized comparison between two networks is shown in Table II.

\section{HARDWARE IMPLEMENTATION}

In this section, we present the proposed architecture for implementation of the neural network that contains two Hopf oscillators, modified astrocyte model, and interconnections for weak coupling and decoupling of the oscillators. The arithmetic structure is shown in Fig. 5(a). In this architecture, two Hopf oscillators are coupled through receiving $x$ and $y$ signals from each other with a specific gain. These coupling connections force the oscillators to remain synchronized. On the other hand, the astrocyte receives $x$ and $y$ signals from both of the oscillators and evaluates and injects a specific signal, $(v)$, for decoupling them. The astrocyte and Hopf blocks contain a digital implementation of the discrete differential equations of the models described in (10) and (11). Each desired signal can be routed to the physical output of target device [in our case, the field-programmable gate array (FPGA)] and converted to analog signal using external digital-to-analog converter chip if it is needed.

\section{A. Hopf Model}

As Fig. 5(b) shows, this unit is a digital implementation of the Hopf oscillator model based on the computational structure of the $X$ and $Y$ equations shown in (21) and (22). Here, we omit the $\omega$-dependent equation of the Hopf oscillator because the oscillation frequency is predetermined. According to the equations, this block receives $x[n], y[n], v[n], x_{c}[n]$, and $y_{c}[n]$ $\left(x_{c}\right.$ and $y_{c}$ are the coupling state signals from the other oscillator) to produce $x[n+1]$ and $y[n+1]$. This process is performed for producing each sample of the outputs using the last samples. In our digital implementation, there is a memory 

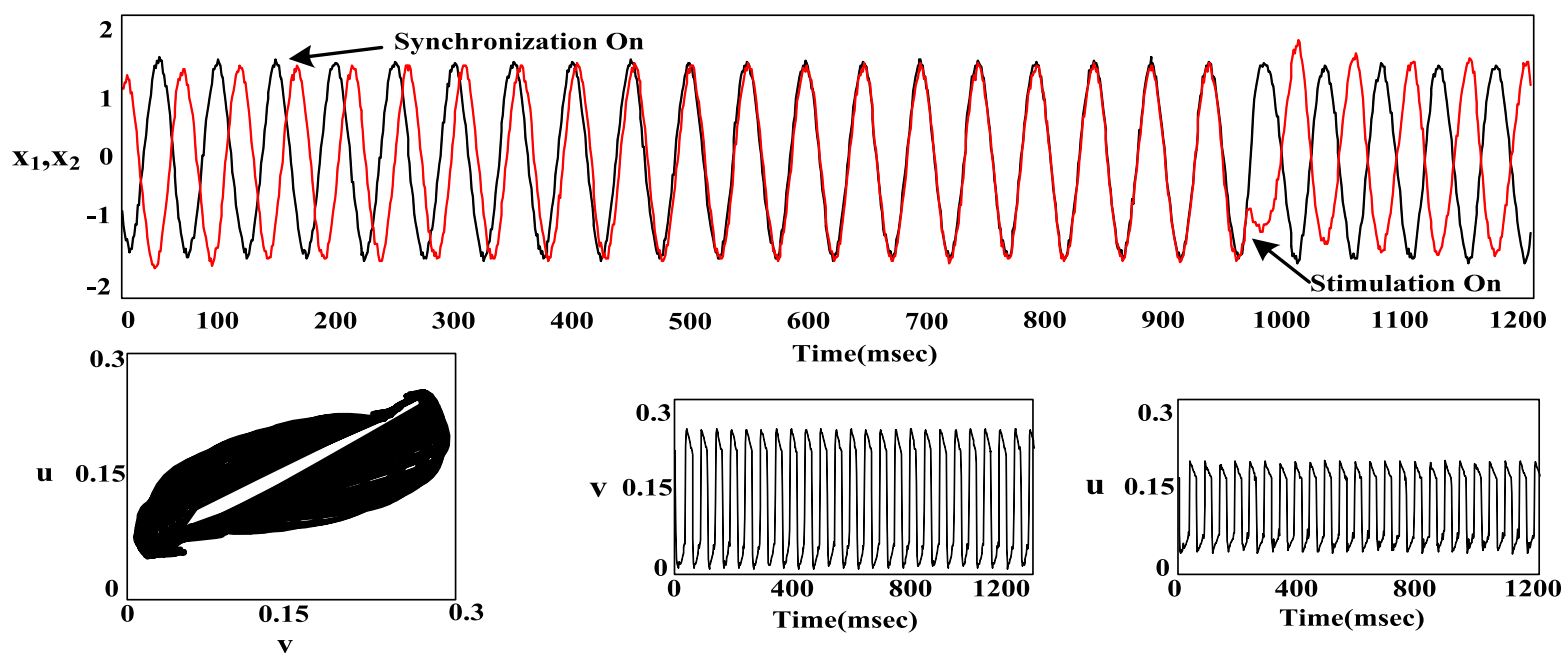

Fig. 6. Digital oscilloscope photographs, which show the effect of varying coupling strength $G$ for the digital implemented neural network on $x_{1}, x_{2}$ for two coupled oscillators with regulating by proposed astrocyte model and phase portrait of modified astrocyte states in $v-u, v$, and $u$ spaces with input $=\sin (25 t)$.

register at the each output of the block that stores the outputs to be used in the next sample calculations through feedback from the memory outputs to the block inputs. Each of the $X$ and $X$ state variables are solved in $X_{b}$ and $Y_{b}$ bits that can be determined according to the target application values range and the required precision for implementation. The multiplied constants are determined in order to be implemented with shift and add operations. For example, the applied sampling time $(d t)$ for digital implementation of the differential equations is equal to $0.0078125=1 / 128=1 /(27)$ that can be implemented by seven arithmetic shifts to the right. Note that this structure can be optimized in area and speed through pipelining and resource sharing, but we implement a single oscillator through a combinational digital circuit for our specific purpose.

\section{B. Modified Astrocyte Model}

This unit contains digital implementation of the modified astrocyte model based on the computational structure of the $v$ and $u$ equations in (10) and (11), as shown in Fig. 5(c). According to the equations, this block receives state signals $x[n]$ and $y[n]$ from both oscillators to produce $u[n+1]$ and $v[n+1]$ and send back $v[n+1]$ to the oscillators to decouple them. Similar to the Hopf model, there is a memory register at the each output of the block that stores the outputs for using in the next sample calculations through a feedback from the memory outputs to the block inputs. Bit numbers of the variables are predetermined according to the application, desired precision, and minimum requirement of the precision in the recursive digital implementation of the differential equations. This structure can be optimized in area and speed through pipelining and resource sharing, but we implement a single astrocyte through a combinational digital circuit in this paper. The modified astrocyte model contains a specific function as $2^{-n} \times K$ (where $n$ is a positive integer number) that is a challenge for combinational digital circuit implementation comparatively. This function can be implemented through $n$ arithmetic shifts to the right on the binary number, $K$. According to the equation, $n$ is a variable number and this implies that we must implement a combinational digital circuit to shift $K$ as determined by input $n$. Fortunately, there is an elegant well-known method for this purpose known as the barrel shifter. This shifter contains one multiplexer for each bit of $n$. Detailed structure of the barrel shifter can be found in the literature.

\section{IMPLEMENTATION RESULTS}

To verify the validity of the proposed astrocyte model and the neuronal network, the case study outlined in Section IV is implemented on a XILINX XUP Virtex-II Pro Development System, which provides a hardware platform that consists of a high performance Virtex-II Pro XC2VP30 Platform FPGA surrounded by a comprehensive collection of peripheral components [30]. Fig. 6 shows digital oscilloscope photographs of the dynamical behaviors of a single proposed astrocyte model implemented on this FPGA platform for $v, u$, and $v-u$ states. High- and low-level device utilization for implementation of a single proposed astrocyte and network are summarized in Tables III and IV.

The results show that the implemented astrocyte model is easily implementable compared with the original model. This is expected because the original model has a term $f\left(c, c_{e}\right)$, $G_{m}$ state variable, and tanh function that would require a high area consumption for a circuit implementation. Moreover, for evaluating performance of the proposed model in a neuronal network for a desynchronization task, the network studied in Section IV is implemented. Hardware synthesis and implementations on FPGA show that the proposed astrocyte model provides appropriate feedback control in regulating the neuronal activities to desynchronization of two coupled limit-cycle oscillators as the same as original model. High- and low-level device utilization for implementation of a single proposed astrocyte and network is summarized in Tables III and IV. 
TABLE III

High-LeVel DeVice Utilization of XILINX VirTeX-II Pro FoR IMPLEMENTATION OF SingLe PRoposed Astrocyte

Model ANd NeURonal NeTwork

\begin{tabular}{ccc}
\hline \hline Resources & $\begin{array}{c}\text { Proposed Astrocyte } \\
\text { Model }\end{array}$ & $\begin{array}{c}\text { Proposed Neuronal } \\
\text { Network }\end{array}$ \\
\hline 30×30-bit multiplier & 1 & 9 \\
30-bit adder & 13 & 17 \\
30-bit subtractor & 2 & 2 \\
31-bit adder & 2 & 2 \\
32-bit adder & 3 & 17 \\
33-bit adder & 1 & 1 \\
35-bit subtractor & 1 & 1 \\
36-bit adder & 1 & 1 \\
55-bit adder carry in & - & 2 \\
55-bit subtractor & - & 2 \\
8-bit adder carry out & 2 & 2 \\
30-bit up accumulator & 2 & 2 \\
Flip-flops & 8 & 128 \\
\hline
\end{tabular}

TABLE IV

Low-LeVel Device Utilization of XiLinX VirTeX-II Pro AND the Used PeRCENTAGe of Basic Elements For IMPLEMENTATION OF Single Proposed Astrocyte Model and Neuronal Network

\begin{tabular}{ccc}
\hline \hline Resources & $\begin{array}{c}\text { Proposed Astrocyte } \\
\text { Model }\end{array}$ & $\begin{array}{c}\text { Proposed Neuronal } \\
\text { Network }\end{array}$ \\
\hline Number of Slices & $189(1.3 \%)$ & $1213(8.8 \%)$ \\
Number of Slice Flip Flops & $68(0.2 \%)$ & $188(0.6 \%)$ \\
Number of 4 input LUTs & $352(1.2 \%)$ & $2321(8.4 \%)$ \\
Number of bonded IOBs & $21(3.7 \%)$ & $22(3.9 \%)$ \\
Number of MULT18X18s & $4(2.9 \%)$ & $36(26 \%)$ \\
Number of GCLKs & $2(12.5 \%)$ & $2(12.5 \%)$ \\
\hline
\end{tabular}

\section{CONCLUSION}

This paper presents a conveniently implementable electronic circuit astrocyte model that exhibits the relevant biological behaviors. Moreover, this lays the groundwork for the investigation of those types of neuronal synchronization that play an important role in biological information processing and some neural system diseases. To this end, a relevant case study is implemented in this paper. Findings show that our proposed modifications simplify the hardware implementation yet demonstrate relevant dynamical behaviors.

\section{APPENDIX A}

\section{Proof of Theorem 1 [26]}

Let $x=0$ be an equilibrium point for the nonlinear system

$$
\dot{x}=f(t, x)
$$

where $f:[0, \infty) \times D \longrightarrow R^{n}$ is continuously differentiable, $D=\left\{x \in R^{n} \mid\|x\|<r\right\}$, and the Jacobian matrix $[\partial f / \partial x]$ is bounded on $D$, uniformly in $t$. Let $k, \lambda$, and $r_{0}$ be positive constants with $r_{0}<r / k$. Let $D_{0}=\left\{x \in R^{n} \mid\|x\|<r_{0}\right\}$. Assume that the trajectories of the system satisfy

$$
\|x(t)\| \leq k\left\|x\left(t_{0}\right)\right\| e^{-\lambda\left(t-t_{0}\right)} \quad \forall x\left(t_{0}\right) \in D_{0} \quad \forall t \geq t_{0} \geq 0 .
$$

Then, there is a function $V:[0, \infty) \times D_{0} \longrightarrow R$ that satisfies the inequalities

$$
\begin{aligned}
b_{1}\|x\|^{2} \leq V(t, x) & \leq b_{2}\|x\|^{2} \\
\frac{\partial V}{\partial t}+\frac{\partial V}{\partial x} f(t, x) & \leq-b_{3}\|x\|^{2} \\
\frac{\partial V}{\partial x} & \leq b_{4}\|x\|
\end{aligned}
$$

for some positive constants $b_{1}, b_{2}, b_{3}$, and $b_{4}$. Moreover, if $r=\infty$ and the origin is globally exponentially stable, then $V(t, x)$ is defined and satisfies the aforementioned inequalities on $R^{n}$. Furthermore, if the system is autonomous, $V$ can be chosen independent of $t$.

Proof [26]: Due to the equivalence of norms, it is sufficient to prove the theorem for the 2-norm. Let $\phi(\tau ; t, x)$ denote the solution of the system that starts at $(t, x)$, that is, $\phi(t ; t, x)=x$. For all $x \in D_{0}, \phi(\tau ; t, x) \in D$ for all $\tau \geq t$. Let

$$
V(x, t)=\int_{t}^{t+\delta} \phi^{T}(\tau ; t, x) \phi(\tau ; t, x) d \tau
$$

where $\delta$ is a positive constant to be chosen. Due to the exponentially decaying bound on the trajectories, we have

$$
\begin{aligned}
V(t, x) & =\int_{t}^{t+\delta}\|\phi(\tau ; t, x)\|_{2}^{2} d \tau \leq \int_{t}^{t+\delta} k^{2} e^{-2 \lambda(\tau-t)} d \tau\|x\|_{2}^{2} \\
& =\frac{k^{2}}{2 \lambda}\left(1-e^{-2 \lambda \delta}\right)\|x\|_{2}^{2}
\end{aligned}
$$

On the other hand, the Jacobian matrix $[\partial f / \partial x]$ is bounded on $D$. Let

$$
\left\|\frac{\partial f}{\partial x}(t, x)\right\|_{2} \leq L \quad \forall x \in D
$$

Then, $\|f(t, x)\|_{2} \leq L\|x\|_{2}$ and $\phi(\tau ; t, x)$ satisfies the lower bound

$$
\|\phi(\tau ; t, x)\|_{2}^{2} \geq\|x\|_{2}^{2} e^{-2 L(\tau-t)} .
$$

Hence

$$
V(t, x) \geq \int_{t}^{t+\delta} e^{-2 L(\tau-t)} d \tau\|x\|_{2}^{2}=\frac{1}{2 L}\left(1-e^{-2 L \delta}\right)\|x\|_{2}^{2} .
$$

Thus, $V(t, x)$ satisfies the first inequality of the theorem with

$$
b_{1}=\frac{\left(1-e^{-2 L \delta}\right)}{2 L}, b_{2}=\frac{k^{2}\left(1-e^{-2 \lambda \delta}\right)}{2 \lambda} .
$$

To calculate the derivative of $V$ along the trajectories of the system, define the sensitivity functions

$\phi_{t}(\tau ; t, x)=\frac{\partial}{\partial t} \phi(\tau ; t, x) ; \phi_{x}(\tau ; t, x)=\frac{\partial}{\partial x} \phi(\tau ; t, x)$. 
Then

$$
\begin{aligned}
& \frac{\partial V}{\partial t}+\frac{\partial V}{\partial x} f(t, x)=\phi^{T}(t+\delta ; t, x) \phi(t+\delta ; t, x) \\
& -\phi^{T}(t ; t, x) \phi(t ; t, x)+\int_{t}^{t+\delta} 2 \phi^{T}(\tau ; t, x) \phi_{t}(\tau ; t, x) d \tau \\
& \quad+\int_{t}^{t+\delta} 2 \phi^{T}(\tau ; t, x) \phi_{x}(\tau ; t, x) d \tau f(t, x) \\
& =\phi^{T}(t+\delta ; t, x) \phi(t+\delta ; t, x)-\|x\|_{2}^{2} \\
& \quad+\int_{t}^{t+\delta} 2 \phi^{T}(\tau ; t, x)\left[\phi_{t}(\tau ; t, x)+\phi_{x}(\tau ; t, x) f(t, x)\right] d \tau .
\end{aligned}
$$

It is shown that

$$
\phi_{t}(\tau ; t, x)+\phi_{x}(\tau ; t, x) f(t, x) \equiv 0 \quad \forall \tau \geq t .
$$

Therefore

$$
\begin{aligned}
\frac{\partial V}{\partial t}+\frac{\partial V}{\partial x} f(t, x) & =\phi^{T}(t+\delta ; t, x) \phi(t+\delta ; t, x)-\|x\|_{2}^{2} \\
& \leq-\left(1-k^{2} e^{-2 \lambda \delta}\right)\|x\|_{2}^{2} .
\end{aligned}
$$

By choosing $\delta=\ln \left(2 k^{2}\right) /(2 \lambda)$, the second inequality of the theorem is satisfied with $b_{3}=1 / 2$. To show the last inequality, let us note that $\phi(\tau ; t, x)$ satisfies the sensitivity equation

$$
\frac{\partial}{\partial \tau} \phi_{x}=\frac{\partial f}{\partial x}(\tau, \phi(\tau ; t, x)) \phi_{x}, \quad \phi_{x}(t ; t, x)=I .
$$

Since

$$
\left\|\frac{\partial f}{\partial x}(t, x)\right\|_{2} \leq L
$$

on $D, \phi_{x}$ satisfies the bound

$$
\left\|\phi_{x}(\tau ; t, x)\right\|_{2} \leq e^{L(\tau-t)} .
$$

Therefore

$$
\begin{aligned}
\left\|\frac{\partial V}{\partial x}\right\|_{2} & =\left\|\int_{t}^{t+\delta} 2 \phi^{T}(\tau ; t, x) \phi_{x}(\tau ; t, x) d \tau\right\|_{2} \\
& \leq \int_{t}^{t+\delta} 2\|\phi(\tau ; t, x)\|_{2}\left\|\phi_{x}(\tau ; t, x)\right\|_{2} d \tau \\
& \leq \int_{t}^{t+\delta} 2 k e^{-\lambda(\tau-t)} e^{L(\tau-t)} d \tau\|x\|_{2} \\
& =\frac{2 k}{(\lambda-L)}\left[1-e^{-(\lambda-L) \delta}\right]\|x\|_{2} .
\end{aligned}
$$

Thus, the last inequality of the theorem is satisfied with

$$
b_{4}=\frac{2 k}{(\lambda-L)}\left[1-e^{-(\lambda-L) \delta}\right] .
$$

If all the assumptions hold globally, then clearly $r_{0}$ can be chosen arbitrarily large. If the system is autonomous, then $\phi(\tau ; t, x)$ depends only on $(\tau-t)$

$$
\phi(\tau ; t, x)=\varphi(\tau-t ; x) .
$$

Then

$$
\begin{aligned}
V(t, x) & =\int_{t}^{t+\delta} \varphi^{T}(\tau-t ; x) \varphi(\tau-t ; x) d \tau \\
& =\int_{0}^{\delta} \varphi^{T}(s ; x) \varphi(s ; x) d s
\end{aligned}
$$

which is independent of $t$. We know that if the linearization of a nonlinear system about the origin has an exponentially stable equilibrium point, then the origin is an exponentially stable equilibrium point for the nonlinear system. We will use Theorem 1 to prove that exponential stability of the linearization is a necessary and sufficient condition for exponential stability of the origin.

\section{APPENDIX B}

\section{PROOF OF THEOREM 2 [26]}

Let $D \subset R^{n}$ be a domain that contains the origin and $V$ : $[0, \infty) \times D \longrightarrow R$ be a continuously differentiable function such that

$$
\begin{gathered}
\alpha_{1}(\|x\|) \leq V(t, x) \leq \alpha_{2}(\|x\|) \\
\frac{\partial V}{\partial t}+\frac{\partial V}{\partial x} f(t, x) \leq-W_{3}(x) \quad \forall\|x\| \geq \mu>0
\end{gathered}
$$

$\forall t \geq 0$ and $\forall x \in D$, where $\alpha_{1}$ and $\alpha_{2}$ are $\kappa$ functions and $W_{3}(x)$ is a continuous positive definite function. Take $r>0$ such that $B_{r} \subset D$ and suppose that

$$
\mu<\alpha_{2}^{-1}\left(\alpha_{1}(r)\right) .
$$

Then, there exists a class of $\kappa \varsigma$ function $\beta$ and for every initial state $x\left(t_{0}\right)$, satisfying $\left\|x\left(t_{0}\right)\right\| \leq \alpha_{2}^{-1}\left(\alpha_{1}(r)\right)$, there is $T \geq 0$ [dependent on $x\left(t_{0}\right)$ and $\mu$ ] such that the solution of $\dot{x}=f(t, x)$ satisfies

$$
\begin{aligned}
& \|x(t)\| \leq \beta\left(\left\|x\left(t_{0}\right)\right\|, t-t_{0}\right) \quad \forall t_{0} \leq t \leq t_{0}+T \\
& \|x(t)\| \leq \alpha_{1}^{-1}\left(\alpha_{2}(\mu)\right) \quad \forall t \leq t_{0}+T .
\end{aligned}
$$

Moreover, if $D=R^{n}$ and $\alpha_{1}$ belongs to class $\kappa_{\infty}$, then (83) and (84) hold for any initial state $x\left(t_{0}\right)$, with no restriction on how large $\mu$ is.

Proof [26]: Inequalities (83) and (84) show that $x(t)$ is uniformly bounded for all $t \geq t_{0}$ and uniformly ultimately bounded with the ultimate bound $\alpha_{1}^{-1}\left(\alpha_{2}(\mu)\right)$. The ultimate bound is a class $\kappa$ function of $\mu$; hence, the smaller the value of $\mu$, the smaller the ultimate bound. As $\mu \longrightarrow 0$, the ultimate bound approaches zero.

\section{APPENDIX C}

PROOF OF THE THEOREM 3 [26]

Let $V:[0, \infty) \times R^{n} \longrightarrow R$ be a continuously differentiable function such that

$$
\begin{aligned}
\alpha_{1}(\|x\|) & \leq V(t, x) \leq \alpha_{2}(\|x\|) \\
\frac{\partial V}{\partial t}+\frac{\partial V}{\partial x} f(t, x, u) & \leq-W_{3}(x) \quad \forall\|x\| \geq \rho(\|u\|)>0
\end{aligned}
$$


$\forall(t, x, u) \in[0, \infty) \times R^{n} \times R^{m}$, where $\alpha_{1}$ and $\alpha_{2}$ are class $\kappa_{\infty}$ functions, $\rho$ is a class $\kappa$ function, and $W_{3}(x)$ is a continuous positive definite function on $R^{n}$. Then, the system $\dot{x}=f(t, x, u)$ is input-to-state stable with $\gamma=\alpha_{1}^{-1} \alpha_{2} \rho$.

Proof [26]: By applying the global version of Theorem 2, we find that the solution $x(t)$ exist and satisfies

$$
\|x(t)\| \leq \beta\left(\left\|x\left(t_{0}\right)\right\|, t-t_{0}\right)+\gamma\left(\sup _{\tau \geq t_{0}}\|u(\tau)\|\right) \quad \forall t \geq t_{0} .
$$

Since $x(t)$ depends only on $u(\tau)$ for $t_{0} \leq \tau \leq t$, the supremum on the right-hand side of (80) can be taken over $\left[t_{0}, t\right]$, which yields

$$
\|x(t)\| \leq \beta\left(\left\|x\left(t_{0}\right)\right\|, t-t_{0}\right)+\gamma\left(\sup _{t_{0} \leq \tau \leq t}\|u(\tau)\|\right) .
$$

\section{REFERENCES}

[1] M. J. Pearson et al., "Implementing spiking neural networks for real-time signal-processing and control applications: A model-validated FPGA approach," IEEE Trans. Neural Netw., vol. 18, no. 5, pp. 1472-1487, Sep. 2007.

[2] S. Hashimoto and H. Torikai, "A novel hybrid spiking neuron: Bifurcations, responses, and on-chip learning," IEEE Trans. Circuits Syst. I, Reg. Papers, vol. 57, no. 8, pp. 2168-2181, Aug. 2010.

[3] T. Hishiki and H. Torikai, "A novel rotate-and-fire digital spiking neuron and its neuron-like bifurcations and responses," IEEE Trans. Neural Netw., vol. 22, no. 5, pp. 752-767, May 2011.

[4] H. Soleimani, A. Ahmadi, and M. Bavandpour, "Biologically inspired spiking neurons: Piecewise linear models and digital implementation," IEEE Trans. Circuits Syst. I, Reg. Papers, vol. 59, no. 12, pp. 2991-3004, Dec. 2012.

[5] L. Geretti and A. Abramo, "The correspondence between deterministic and stochastic digital neurons: Analysis and methodology," IEEE Trans. Neural Netw., vol. 19, no. 10, pp. 1739-1752, Oct. 2008.

[6] J. Zhou, W. Yu, X. Li, M. Small, and J. A. Lu, "Identifying the topology of a coupled FitzHugh-Nagumo neurobiological network via a pinning mechanism," IEEE Trans. Neural Netw., vol. 20, no. 10, pp. 1679-1684, Oct. 2009

[7] G. Indiveri, E. Chicca, and R. Douglas, "A VLSI array of low-power spiking neurons and bistable synapses with spike-timing dependent plasticity," IEEE Trans. Neural Netw., vol. 17, no. 1, pp. 211-221, Jan. 2006.

[8] R. Serrano-Gotarredona et al., "CAVIAR: A $45 \mathrm{k}$ neuron, 5M synapse, $12 \mathrm{G}$ connects/s AER hardware sensory-processing-learningactuating system for high-speed visual object recognition and tracking," IEEE Trans. Neural Netw., vol. 20, no. 9, pp. 1417-1438, Sep. 2009.

[9] R. J. Vogelstein, U. Mallik, J. T. Vogelstein, and G. Cauwenberghs, "Dynamically reconfigurable silicon array of spiking neurons with conductance-based synapses," IEEE Trans. Neural Netw., vol. 18, no. 1, pp. 253-265, Jan. 2007.

[10] R. Serrano-Gotarredona, T. Serrano-Gotarredona, A. Acosta-Jiménez, and B. Linares-Barranco, "A neuromorphic cortical-layer microchip for spike-based event processing vision systems," IEEE Trans. Circuits Syst. I, Reg. Papers, vol. 53, no. 12, pp. 2548-2566, Dec. 2006.

[11] (2013, Dec.). Blue Brain Project [Online]. Available: http://bluebrain.epfl.ch

[12] S. B. Furber, S. Temple, and A. D. Brown, "High-performance computing for systems of spiking neurons," in Proc. AISB Workshop GC5, Archit. Brain Mind, vol. 2. 2006, pp. 29-36.

[13] M. V. Sofroniew and H. V. Vinters, "Astrocytes: Biology and pathology," Acta Neuropathologica, vol. 119, no. 1, pp. 7-35, 2010.

[14] M. Nedergaard, B. Ransom, and S. A. Goldman, "New roles for astrocytes: Redefining the functional architecture of the brain," Trends Neurosci., vol. 26, no. 10, pp. 523-530, 2003.

[15] P. G. Haydon, "Glia: Listening and talking to the synapse," Nature Rev., vol. 2, no. 1, pp. 185-193, 2001.

[16] R. D. Fields and B. Stevens-Graham, "New insights into neuron-glia communication," Science, vol. 298, no. 5593, pp. 556-562, 2002.

[17] G. Bonvento, C. Giaume, and J. Lorenceau, "Neuron-glia interactions: From physiology to behavior," J. Physiol. Paris, vol. 96, no. 3, pp. $167-168,2002$.
[18] S. Nadkarni and P. Jung, "Dressed neurons: Modeling neural-glial interactions," Phys. Biol., vol. 1, no. 1, pp. 35-41, 2004.

[19] S. Nadkarni and P. Jung, "Modeling synaptic transmission of the tripartite synapse," Phys. Biol., vol. 4, no. 1, pp. 1-9, 2007.

[20] S. Nadkarni, P. Jung, and H. Levine, "Astrocytes optimize the synaptic transmission of information," PLoS Comput. Biol., vol. 4, no. 5, pp. 1-9, 2008.

[21] D. E. Postnov, L. S. Ryazanova, and O. V. Sosnovtseva, "Functional modeling of neural-glial interaction," BioSystems, vol. 89, no. 1 , pp. 84-91, 2007.

[22] D. E. Postnov, R. N. Koreshkov, N. A. Brazhe, A. R. Brazhe, and O. V. Sosnovtseva, "Dynamical patterns of calcium signaling in a functional model of neuron-astrocyte networks," Biol. Phys., vol. 35, no. 4, pp. 425-445, 2009

[23] C. A. S. Batista, S. R. Lopes, R. L. Viana, and A. M. Batista, "Delayed feedback control of bursting synchronization in a scale-free neuronal network," Neural Netw., vol. 23, no. 1, pp. 114-124, 2010.

[24] G. Montaseri, M. J. Yazdanpanah, and M. Amiri, "Astrocyte-inspired controller design for desynchronization of two coupled limit-cycle oscillators," in Proc. IEEE 3rd World Congr. NaBIC, Oct. 2011, pp. 195-200.

[25] S. Marra, M. A. Iachino, and F. C. Morabito, "Tanh-like activation function implementation for high-performance digital neural systems," in Proc. 2nd Conf. PhD Res. Microelectron. Electron., Otranto, Italy, Jun. 2006, pp. 237-240.

[26] H. K. Khalil, Nonlinear Systems, vol. 3. Upper Saddle River, NJ, USA: Prentice-Hall, 2002

[27] D. V. Reddy, A. Sen, and G. L. Johnston, "Dynamics of a limit cycle oscillator under time delayed linear and nonlinear feedbacks," Phys. D. Nonlinear Phenomena, vol. 144, no. 3, pp. 335-357, 2000.

[28] P. F. Pinsky and J. Rinzel, "Synchrony measures for biological neural networks," Biol. Cybern., vol. 73, no. 2, pp. 129-137, 1995.

[29] L. Gray, "A mathematician looks at Wolfram's new kind of science," Notices Amer. Math. Soc., vol. 50, no. 2, pp. 200-211, 2003.

[30] Xilinx, Inc., San Jose, CA, USA. (2013, Mar.). FPGA and HDL Software Package [Online]. Available: http://www.xilinx.com/

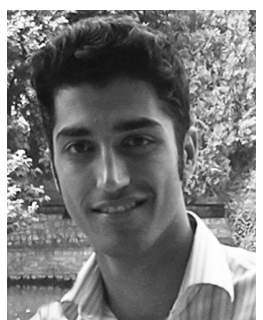

Hamid Soleimani received the B.Sc. and M.Sc. degrees in electronic engineering from Razi University, Kermanshah, Iran, in 2010 and 2012, respectively.

He is currently a Researcher with the Karin Cognitive Center, Kermanshah. His current research interests include digital and analog circuit design and optimization for neuromorphic systems, neurosynaptic architecture, dynamical systems, and parallel processing.

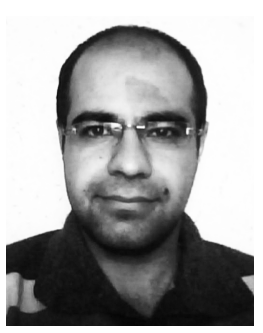

Mohammad Bavandpour received the B.Sc. and M.Sc. degrees in electronic engineering from Razi University, Kermanshah, Iran, and the Sharif University of Technology, Tehran, Iran, in 2010 and 2012, respectively

He is currently a Researcher with the Karin Cognitive Center, Kermanshah. His current research interests include brain simulation, dynamical systems, memristive systems, digital circuit design and optimization, artificial intelligence, and parallel processing.

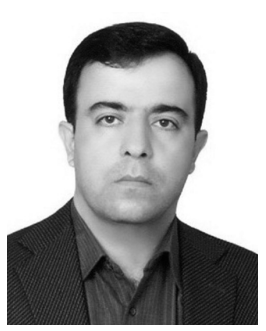

Arash Ahmadi (M'04) received the B.Sc. and M.Sc. degrees in electronic engineering from the Sharif University of Technology, Tehran, Iran, and Tarbiat Modares University, Tehran, respectively, and the $\mathrm{Ph} . \mathrm{D}$. degree in electronics from the University of Southampton, Southampton, U.K., in 1993, 1997, and 2008, respectively.

He has been with Razi University, Kermanshah, Iran, since 1997, as a Faculty Member. From 2008 to 2010, he was a Fellow Researcher with the University of Southampton. He is currently an Assistant Professor with the Electrical Engineering Department, Razi University. His current research interests include hardware implementation of signal processing systems, high-level synthesis, neuromorphic systems, neurosynaptic architecture, and bioinspired computing. 


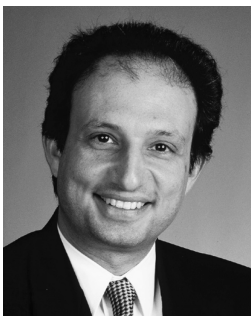

Derek Abbott (M'85-SM'99-F'05) was born in South Kensington, London, U.K., in 1960. He received the B.S. (Hons.) degree in physics from the Loughborough University, Leicestershire, U.K., and the $\mathrm{Ph} . \mathrm{D}$. degree in electrical and electronic engineering under K. Eshraghian and B. R. Davis from the University of Adelaide, Adelaide, SA, Australia, in 1982 and 1995, respectively.

He was with the GEC Hirst Research Centre, London, U.K., from 1978 to 1986, and Austek Microsystems, Technology Park, SA, Australia, in 1986. Since 1987, he has been with the University of Adelaide, where he is currently a Full Professor with the School of Electrical and Electronic Engineering. He has appeared on national and international television and radio and has also received scientific reportage in New Scientist, The Sciences, Scientific American, Nature, The New York Times, and Sciences et Avenir. He holds more than 800 publications/patents and has been an Invited Speaker at more than 100 institutions, including the Princeton University, NJ, USA, the MIT, MA, USA, the Santa Fe Institute, NM, USA, the Los Alamos National Laboratories, NM, USA, the Cambridge University, Cambridge, U.K., and the École Polytechnique Fédérale de Lausanne, Lausanne, Switzerland. He has co-edited Quantum Aspects of Life (Imperial College Press, 2007), co-authored Stochastic Resonance (Cambridge University Press, 2008), and co-authored Terahertz Imaging for Biomedical Applications (Springer, 2012).

Prof. Abbott is a fellow of the Institute of Physics (IOP). He is a recipient of the GEC Bursary in 1977, the Stephen Cole Elder Prize in 1998, the SPIE Scholarship Award for Optical Engineering and Science in 2003, the South Australian Tall Poppy Award for Science in 2004, and the Premier's SA Great Award in Science and Technology for outstanding contributions to South Australia in 2004. He was awarded a prestigious Australian Research Council (ARC) Future Fellowship at the highest level in 2012. He has served as an Editor and/or Guest Editor for a number of journals, including IEEE JOURNAL OF SOLID-STATE CIRCUITS, Chaos (AIP), Smart Structures and Materials (IOP), the Journal of Optics B (IOP), the Microelectronics Journal (Elsevier), andFluctuation Noise Letters (World Scientific), and is currently on the Editorial Boards of the PROCEEDINGS OF THE IEEE, the IEEE Photonics JOURNAL, and PLOSONE. He has served on a number of IEEE technical program committees, including the IEEE Asia-Pacific Conference on Circuits and Systems and the IEEE GaAs IC Symposium 